\title{
Efficient Topology-Aware Overlay Network
}

\author{
Marcel Waldvogel \\ mwl@zurich.ibm.com
}

\author{
Roberto Rinaldi \\ rob_rinaldi@virgilio.it
}

\author{
IBM Research \\ Zurich Research Laboratory \\ Säumerstrasse 4 / Postfach \\ 8803 Rüschlikon, Switzerland
}

\begin{abstract}
Peer-to-peer (P2P) networking has become a household word in the past few years, being marketed as a work-around for server scalability problems and "wonder drug" to achieve resilience. Current widely-used $\mathrm{P} 2 \mathrm{P}$ networks rely on central directory servers or massive message flooding, clearly not scalable solutions. Distributed Hash Tables (DHT) are expected to eliminate flooding and central servers, but can require many long-haul message deliveries. We introduce Mithos, an content-addressable overlay network that only uses minimal routing information and is directly suitable as an underlay network for $\mathrm{P} 2 \mathrm{P}$ systems, both using traditional and DHT addressing. Unlike other schemes, it also efficiently provides locality-aware connectivity, thereby ensuring that a message reaches its destination with minimal overhead. Mithos provides for highly efficient forwarding, making it suitable for use in high-throughput applications. Paired with its ability to have addresses directly mapped into a subspace of the IPv6 address space, it provides a potential candidate for native deployment. Additionally, Mithos can be used to support third-party triangulation to quickly select a close-by replica of data or services.
\end{abstract}

\section{INTRODUCTION}

The computing world is experiencing a transition from fixed servers and stationary desktop PCs to connected information appliances and ubiquitous connectivity, profoundly changing the way we use information. With cellular data communication, Bluetooth, and IEEE 802.11b (WiFi), the need for a global system that supports these new communication patterns becomes more pressing day by day. Three main patterns can be identified: First, Internet routing table size is surging, second, Internet protocol (IP) forwarding is still a bottleneck in routers, and third, direct serverless communication is gaining importance.
To be presented at Hot Topics in Networks (HotNets-I), October 28-29, 2002, Princeton, NJ, USA. Scheduled to appear in ACM Computer Communication Review (CCR), 33(1), January 2003.
Routing Table Size. The ever increasing size of the Internet routing tables calls for new ways in network protocols. Although the introduction of Classless InterDomain Routing (CIDR) [1] enabled large-scale aggregation of routing information and thus provided a respite in the exponential growth of routing and forwarding tables for several years, the expansion has resumed in the first half of 2001 with full strength. Among the reasons given for the increased growth rates are the exhausting of preallocated address ranges, proliferation of always-on connected devices, and, probably most significantly, the tendency for businesses and even small Internet Service Providers (ISPs) to become multi-homed. This fact of being connected to multiple upstream providers breaks the hierarchy model behind CIDR, which is necessary for its aggregation to be efficient.

Forwarding Lookups. In the early Internet days, packet forwarding was done by a single hash or index table lookup. With the introduction of CIDR to keep routing table size under control, a more complex lookup was required, performing a longest prefix match, which has long been an obstacle to building fast routers serving high-speed links. Novel algorithms [2-4] as well as additional protocol layers such as MPLS [5] have reduced the cost of prefix matching. Any new network design aiming for high data rates should provide for inexpensive lookups.

Symmetric, Serverless Communication. While services such as Napster brought publicity to the term peerto-peer (P2P), serverless communication only started becoming popular when Napster's demise became a possibility. The events of September 11, 2001, have further shown that centralized servers and thus single points of failure should be avoided when system reliability and availability are business-critical. Serverless systems of the first generation heavily relied on flooding as the prime mechanism to query the distributed directory and to support connectivity when network components become unavailable. The second generation being designed now is based on distributed hash tables (DHTs) to allow direct addressing once the ID of the resource, such as document or service, is known. 
Although many theoretical schemes for minimizing routing information have been proposed and many designs for DHTs have recently become prominent discussion topics, we are unaware of any practical and efficient system combining both. In this paper, we introduce Mithos, a novel mechanism that combines both, and provides additional benefits, such as its ability to use IPv6 as a native transport mechanism and its support for third-party triangulation.

Unlike other systems that map Internet topology to Cartesian coordinates [6,7], Mithos, in full P2P spirit, uses every node in the entire network also as a topology landmark. This helps achieve accuracy and efficiency without the overhead of numerous dimensions or full-mesh probing of all landmarks. Instead, directed incremental probing is used to find a near-optimal placement, as will be explained below.

In Mithos, routing table size is minimized because every node only needs to know its direct neighbors; transitive routing enables messages to reach any destination nevertheless. To achieve this, Mithos employs a novel approach to routing in multi-dimensional irregular meshes, which is key to achieving minimum routing table size while guaranteeing connectivity.

The remainder of the paper is organized as follows. Section 2 introduces and describes the concepts behind Mithos. Section 3 presents early results from our simulation environment. Related work is discussed in Section 4, and conclusions are drawn in Section 5.

\section{MITHOS DESIGN}

The basic idea of Mithos is to embed the network into a multi-dimensional space, with every node being assigned a unique coordinate in this space. This is similar to interconnects used in many high-performance parallel computers, enabling optimal global routing with simple knowledge of the local coordinate gradients, i.e., which links lead to higher/lower coordinates in which dimensions. Unlike parallel computers, however, the mesh used for Mithos connectivity is not regular, in order to accommodate dynamic membership as well as to represent locality.

These goals are established for every new node in a threephase process:

1. Finding close-by nodes and establishing a neighborhood

2. Assigning an ID to the newcomer based on this neighborhood

3. Establishing links with the neighborhood

The individual phases are discussed in more detail below.

\subsection{Finding Neighbors}

To ensure that neighbors in the overlay network are also close in the "underlay" network, a distance metric and a location process need to be defined. We chose network delay between two nodes as metric for measuring distances, but any metric establishing geometry-like foundations would

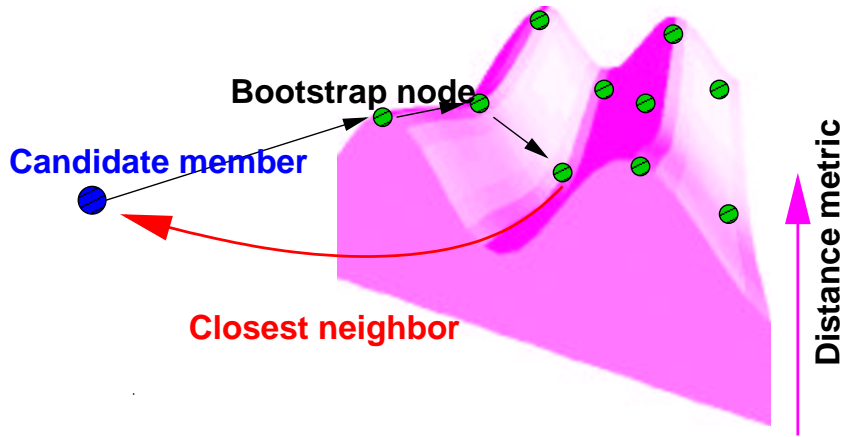

Figure 1: Finding neighbors

be suitable, including any metrics typically used in routing protocols, independent of their Quality-of-Service (QoS) awareness. Examples include physical distance, monetary link cost, or the bandwidth a TCP-compliant stream would achieve. ${ }^{1}$ Independent of the metric used, the value is referred to as distance below.

It is well known that connectivity and connection parameters are not necessarily symmetric or transitive in the Internet, especially when multiple autonomous systems (AS) are involved [8]. Nevertheless, these metrics provide a reasonable basis for an overlay network. When setting up a sufficiently dense overlay network whose goal is to minimize these connection parameters on a per-link basis, the overlay will adapt itself, trying to get optimal service from the underlay.

When searching for neighbors, the natural choice would be to perform an expanding ring search using a multicast mechanism [9]. Although the protocols were defined more than a decade [10], multicast is still only available as an experimental platform in the Internet, if at all. Therefore, the neighborhood location process has to revert to using unicast.

For bootstrapping, Mithos requires a candidate member to know how to contact (at least) one of the existing members. A nonempty subset of these members is used as the first set of candidate neighbors. Then, knowledge from within the overlay network is used to locate the actual neighborhood as follows. Each candidate neighbor is first asked for its direct neighbors, then these neighbors are probed for their distance according to the metric chosen for the overlay system. The best node is then used as the new candidate neighbor. This process is iterated until no further improvement can be achieved, effectively following the distance gradient (Figure $1)$.

As this process is prone to terminate at a local instead of the global minimum, local minima must be recognized and avoided. For Mithos, this is currently done by probing all nodes that are two steps away from the current minimum

\footnotetext{
${ }^{1}$ When setting up a system, care should be taken that the metric chosen is relatively stable for the duration of the P2P network.
} 
before giving up. If a better candidate neighbor is found, the iterative process continues.

\subsection{ID Assignment}

Now that one of its neighbors has been selected, it is necessary to actually assign an ID to the candidate member. This ID selection process is critical, as an inappropriate assignment will eventually create many local minima, preventing an efficient neighborhood location in the future.

Mithos uses the distances measured during the last step of neighborhood establishment as a basis for ID assignment. The two closest nodes found in the process, their neighbors, and the corresponding distances are used in this computation, which requires no further communication.

For ID calculation, virtual springs are established between the candidate member and its fixed neighbors. The tension of each spring is set to be inversely proportional to the distance measured. Then this virtual equivalent of a physical system is allowed to settle, achieving the minimum energy state. This minimum energy location of the candidate node in the multidimensional space is directly used for its ID.

Now that an ID has been established, distances are computed in ID space, no longer requiring measurements (and thus message exchanges) according to the distance metric.

\subsection{Linking Options}

The final step is the establishment of peering relationships between neighbors. To evaluate the possible options for interconnecting neighbors, we established the following criteria:

1. Minimum routing table size;

2. efficient connectivity, full reachability; and

3. fast and simple forwarding algorithm.

These goals would be readily achieved by the strongly regular hypercube or hypertorus interconnect used in many parallel computers. In the presence of network dynamics, the regularity requirement would need to be significantly weakened. Our criterion of maintaining locality between neighbors completely breaks the dynamic supercomputer analogy. Furthermore, locality can lead to some local clustering effects, which need to be dealt with. Alternatives to rectangular connectivity in dynamic, locality-preserving environments are described and evaluated below.

Closest to axis. Along each axis in each direction, find a node that is closest to this axis and establish a link. Then, use the traditional hypertorus forwarding mechanism when delivering messages.

Quadrant-based. Each node establishes a link to the closest neighbor in each quadrant. ${ }^{2}$ When forwarding, the

${ }^{2}$ We use the term "quadrant" as a generic term, even when the number of dimensions, $d$, does not equal 2. All quadrants are determined relative to the current node.

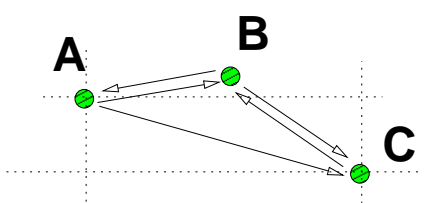

(a)

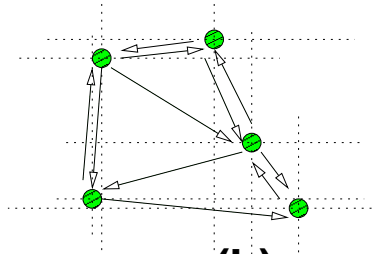

(b)
Figure 2: Example quadrant links in 2-space

next hop is chosen as the neighbor in the same quadrant as the final destination. This can done by computing the difference vector between the current node and the destination, and using the bit vector of the resulting $d$ sign bits (one per dimension) as an index into the next-hop table.

Rectangular subdivision. Each node is assigned an enclosing axis-parallel multi-dimensional rectangle [11]. Forwarding is done to the rectangle abutting at the point where the vector to the destination intersects with the current node's rectangle boundary.

Delaunay triangulation. Establish links according to a Delaunay triangulation of the nodes. Forward analogous to the previous whose vector is angularly closest to the destination vector.

All of these approaches typically achieve small routing tables, although in the worst case (for all but the axis mechanism) a single node could have all other nodes in the system as neighbors.

The connectivity is efficient, except when using closest to axis, which fails to locate off-axis nodes closer than the next on-axis node.

Forwarding lookups are optimal for the quadrants solution, as the final next-hop decision can be made by a simple indexed array access, following a per-dimension subtraction and concentration of sign bits. Many processor architectures offer support for SIMD arithmetic or aggregation of values, as they are easy to implement. Forwarding is still very good for the axis method, but as this method is unable to find all nodes without the aid of another algorithm, we consider it impractical, Rectangles and Delaunay base their decisions on angular calculations and comparisons, requiring expensive multiplications and multidimensional range searches.

We therefore decided to use a quadrant-based mechanism, as it easily fulfilled all the criteria.

\subsection{Establishing Quadrant Links}

Before describing how to achieve quadrant-based links, we first evaluate some of their properties. Figure 2 shows two excerpts of networks situated in 2-space. Looking at Figure 2 (a), even though $\mathrm{A}$ has $\mathrm{C}$ as its closest southeast neighbor, $\mathrm{C}$ does not consider A as its closest northwest neighbor, resulting in asymmetric links. Fortunately, this asymmetry 


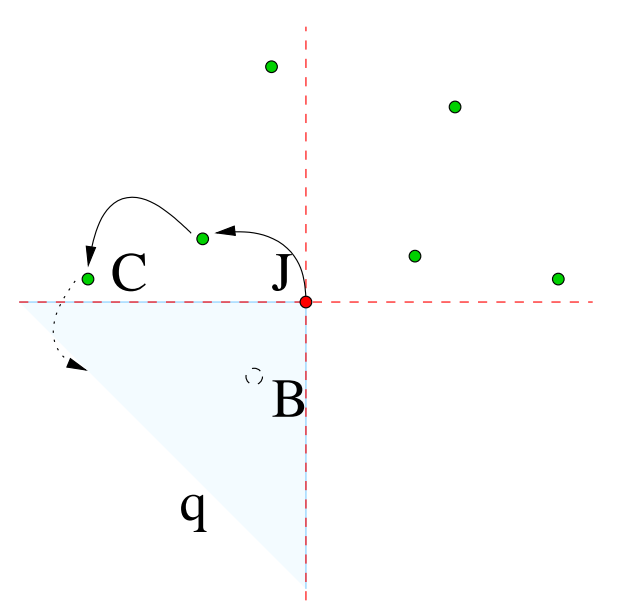

Figure 3: Finding neighbors in all quadrants

has no functional drawbacks during forwarding, as all nodes can still be reached efficiently. However, it needs to be taken into account when establishing the links. To simplify the description, the routing and link establishment process establishes bidirectional links, even though some of them will be used only unidirectionally when forwarding. Thus, the forwarding database remains minimum.

When the joining node $J$ has established its ID, the sum of neighbors that helped it establish its ID may have no information about the best neighbor in all of $J$ 's quadrants. This can be because $J$ 's final position is out of range of the nodes' knowledge, or due to the asymmetry of the routing (cf. Figure 2). Furhermore, even though $J$ might know of a node in each quadrant, this does not necessarily imply that this node is also the node closest to $J$. Therefore, $J$ needs to identify the best neighbors in the region. The mechanism to achieve this is based on ideas similar to the perimeter walk used in Greedy Perimeter Stateless Routing (GPSR) [12], but has been extended to higher dimensions.

Now that a complete neighborhood has been established, it must be ensured that links are established to the closest neighbors, in order to guarantee correct forwarding operation. Thus the second phase tries to locate a closer neighbor by starting at the known neighbor and scanning towards all quadrant borders (Figure 4).

This second phase is an even further generalization of GPSR [12]. It currently uses parallel path processing, which we expect can be optimized further by taking into account further geometric properties of the node relationships. Our early simulations have revealed that in the vast majority of cases, the best neighbors are already known from the merge step. The process is described in more detail in [13].

Serialization of multiple join events is only necessary if they involve the same neighborhood. As the steps requiring serialization all operate only on highly local areas with short distances, serializing them is not expected to become a bottleneck, although we are looking at ways to improve that.

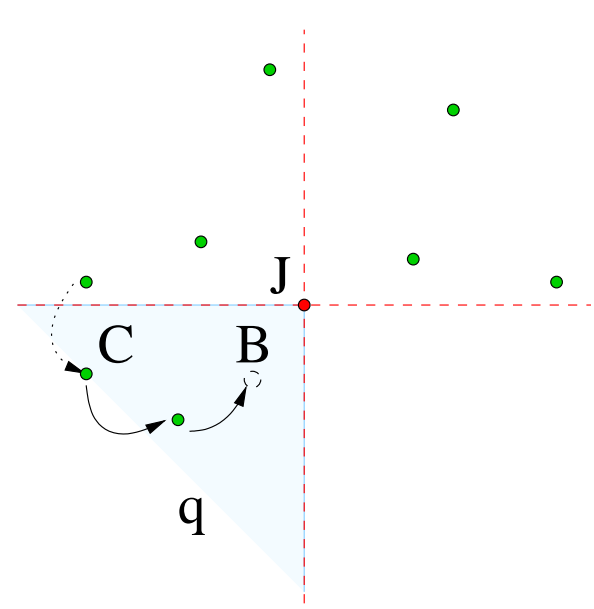

Figure 4: Finding the best neighbor in a quadrant

\subsection{Priming the Overlay Network}

Starting the network from a single node using the mechanisms described above can lead to a very uneven utilization of the available space. To initialize the constants and provide enough initial points required for the spring forces algorithm, the network is primed with a small number of nodes appropriately distributed throughout the space the overlay network should span. These initial nodes are preferentially selected from early nodes interested in joining the system, but we envision that appropriate public landmarks could also be used to bootstrap the system.

\section{RESULTS}

Preliminary results indicate that the above algorithms work very well. Figure 5 shows the quality of the minimumfinding algorithm. Despite its simple heuristics, the results are very encouraging. The test network consisted of 10,000 nodes in the underlay network (generated using the INET topology generator ${ }^{3}$ ) and 1000 nodes in the four-dimensional overlay network. About half of the nodes are optimally placed and more than $90 \%$ of the nodes are less than a factor of 5 in delay from their minimum. Further analysis reveals that this is often due to the small absolute delay.

Figure 6 compares the overhead of end-to-end path lengths under different numbers of dimensions (the same underlay network was used, but this time, only 200 nodes are placed in the overlay network for simulation efficiency). As can be seen, already at four dimensions, more than $97 \%$ of the paths are less than a factor of 3 from optimal. This is in contrast to non-P2P localization algorithms which require more dimensions and do not provide an efficient addressing scheme at the same time.

We expect better placement heuristics to further improve these results at potentially even further savings during node placement. More of our early results can be found in [14].

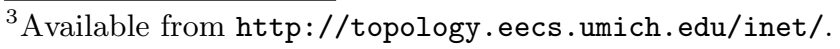




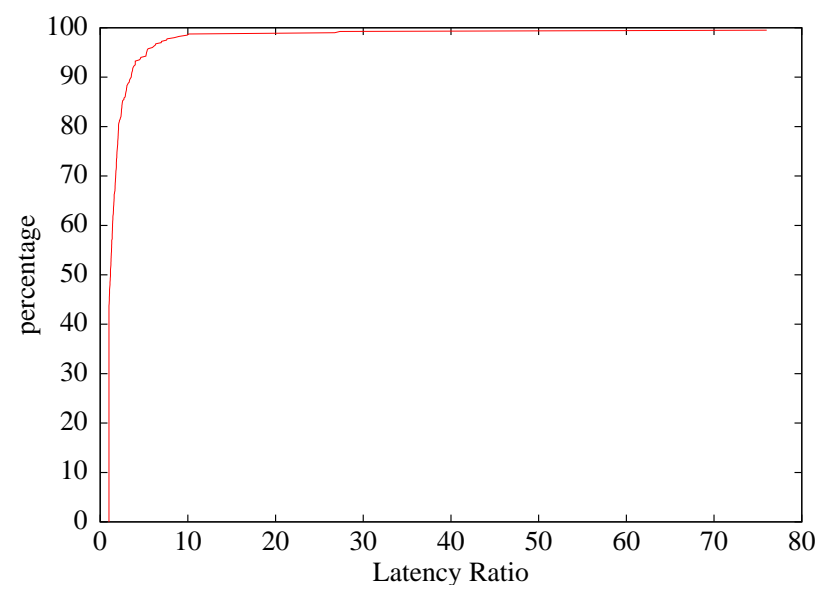

Figure 5: Latency ratio from the local/global minimum for each joining node (CDF)

\section{RELATED WORK}

Cartesian mapping of the Internet has been a topic in the papers by Francis et al. [6] and, more recently, by $\mathrm{Ng}$ and Zhang [7] use landmarks and measurements for triangulation. These two systems rely on a small number of landmarks to provide their measurements. For the system to work, there is thus a critical need for a reliable infrastructure offering these landmarks at high availability. Temporary failure or unreachability of a subset of these nodes will make it hard to compare the proximity of new nodes.

A series of scalable overlay networks have recently sprung to life, such as CAN [15], Chord [16], Pastry [17], and Tapestry [18], all offering a DHT service. The respective locality properties of CAN, Chord, and Pastry are discussed below, separated into geographic layout and proximity forwarding, categories adapted from Castro et al. [19]. ${ }^{4}$

CAN is based on connectivity in a $d$-dimensional space which is subdivided into hypercuboids, which are logically connected along touching surfaces. Initially, CAN's locality was based on proximity forwarding: each node keeps track of the quality of the neighbor links, measured by the ratio of forwarding progress (in the $d$-dimensional space) vs. round-trip time to that neighbor. Later, it was refined to use layout as well, where it adopted a binning scheme [20] to determine neighborhood during node placement. This binning scheme is based upon ranking the relative distances to a given set of landmarks as well as the absolute distances, the latter having been heavily quantized before being used for comparisons. A newly joining node is then placed close to an existing node with a similar landmark triangulation.

Chord extends on the ideas of interval routing [21] by providing for dynamic behavior and proximity forwarding. All nodes are arranged on a conceptual circle, with each node having forwarding fingers (chords) to various other places

\footnotetext{
${ }^{4}$ Tapestry does not directly take advantage of locality itself, due to the strong similarity of the routing mechanism to Pastry, the observations discussed below equally apply to both.
}

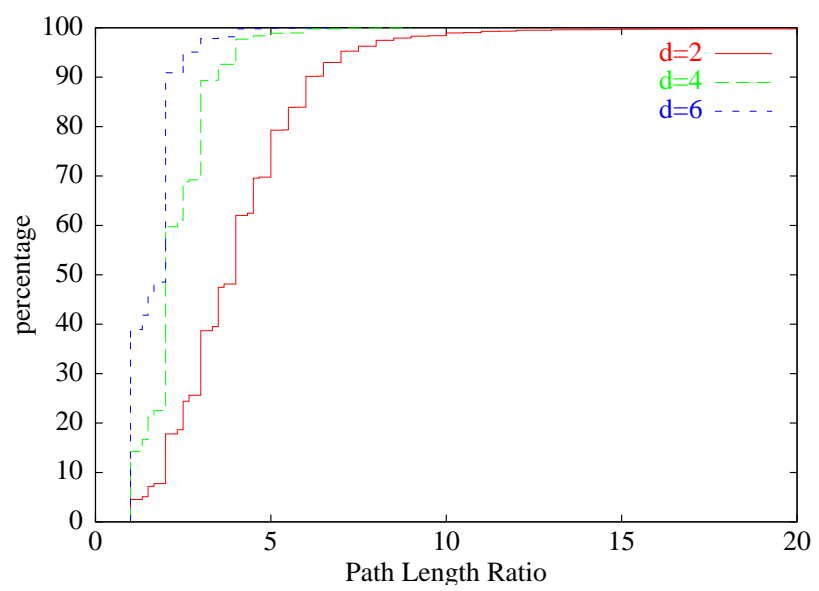

Figure 6: Path length ratios with 2, 4, and 6 dimensions (CDF)

along the circle. These fingers are constantly refined to point to nodes in close proximity, which can lead to significant improvements in forwarding.

Pastry (and Tapestry) routing is similar to radix tries. A message reaches a destination by continuously following to a node with a longer shared prefix between the destination and next-hop IDs. Despite being based on a tree structure, there is no central point of failure, as every participant is both a root, a leaf, and a set of interior nodes in a cleverly interwoven set of tries. Again, proximity forwarding is chosen to take advantage of locality. Among the nodes eligible as children of a particular tree node, the closest node known is picked. According to Castro et al. [19], this allows for a child choice from a much larger set than possible with Chord, resulting in shorter paths.

Among the DHTs, CAN is closest to Mithos in terms of features provided, but uses an entirely different approach; nevertheless, we expect the reliance on a small subset of landmarks, the coarse binning scheme, and the weak integration between layout and routing to provide a performance disadvantage.

\section{CONCLUSIONS AND FUTURE WORK}

By having all nodes in the P2P overlay network provide neighborhood location service through a directed, efficient search, we are able to create an overlay network whose connectivity is close to the optimum achievable with full topology knowledge. In contrast to other approaches, Mithos does not require full topology knowledge, even the forwarding and routing information is minimum and can be used in a highly efficient manner. At the same time, Mithos provides a close conceptual integration between geographic layout and proximity routing, as well as a powerful addressing scheme directly suitable for use in DHTs.

Another key distinguishing factor to both overlay networks as well as the underlying Internet protocol (IP) is the efficiency of the forwarding lookup: its next-hop calculation requires only a few fast processor instructions (or simple 
hardware) and a single indexed memory lookup, significantly faster than comparable or even less feature-rich systems. We believe that such addresses could be directly used in a native, dedicated subspace of the IP version 6 address space [22] to provide efficient addressing and forwarding, e.g., by using six dimensions of 16 bit resolution each.

In the future, we will investigate the dynamic behavior of the network and how to handle asymmetric underlay failures. We also plan to employ metrics obtained from real networks, including metrics other than pure delay. Further topics include optimizations of the "local minimum" and "spring forces" heuristics, as well as evaluating "asymmetric" dimensions, such as local and non-wrapping dimensions, which we expect to be useful when dealing with non-uniform address space usage, but also will provide significant gains for improving locality.

\section{REFERENCES}

[1] Vince Fuller, Tony Li, Jessica Yu, and Kannan Varadhan. Classless Inter-Domain Routing (CIDR): An address assignment and aggregation strategy. Internet RFC 1519, September 1993.

[2] Mikael Degermark, Andrej Brodnik, Svante Carlsson, and Stephen Pink. Small forwarding tables for fast routing lookups. In Proceedings of ACM SIGCOMM, pages 3-14, September 1997.

[3] Marcel Waldvogel, George Varghese, Jon Turner, and Bernhard Plattner. Scalable high speed IP routing table lookups. In Proceedings of ACM SIGCOMM, pages 25-36, September 1997.

[4] Butler Lampson, V. Srinivasan, and George Varghese. IP lookups using multiway and multicolumn search. In Proceedings of IEEE INFOCOM, San Francisco, 1998.

[5] E. C. Rosen, A. Viswanathan, and R. Callon. Multiprotocol label switching architecture. RFC 3031, Internet Engineering Task Force, January 2001.

[6] Paul Francis, Sugih Jamin, Vern Paxson, Lixia Zhang, Daniel F. Gryniewicz, and Yixin Jin. An architecture for a global Internet host distance estimation service. In Proceedings of IEEE INFOCOM, pages 210-217, New York, NY, USA, March 1999.

[7] T. S. Eugene Ng and Hui Zhang. Predicting Internet network distance with coordinates-based approaches. In Proceedings of IEEE INFOCOM, pages 170-179, New York, NY, USA, June 2002.

[8] Stefan Savage et al. Detour: A case for informed Internet routing and transport. IEEE Micro, 19(1):50-59, January 1999.

[9] Sally Floyd, Van Jacobson, Steve McCanne, Lixia Zhang, and Ching-Gung Liu. A reliable multicast framework for light-weight sessions and application level framing. In Proceedings of ACM SIGCOMM, pages 342-356, September 1995.
[10] Stephen Deering and David R. Cheriton. Multicast routing in datagram internetworks and extended LANs. ACM Transactions on Computer Systems, 8(2):85-110, May 1990.

[11] Sylvia Ratnasamy, Scott Shenker, and Ion Stoica. Routing algorithms for DHTs: Some open questions. In Proceedings of First International Workshop on Peer-to-Peer Systems (IPTPS), 2002.

[12] Brad Karp and H. T. Kung. GPSR: Greedy perimeter stateless routing for wireless networks. In Proceedings of MobiCom, pages 243-254, August 2000.

[13] Roberto Rinaldi. Routing and data location in overlay peer-to-peer networks. Diploma thesis, Institut Eurécom and Università degli Studi di Milano, June 2002. Also available as IBM Research Report RZ-3433.

[14] Roberto Rinaldi and Marcel Waldvogel. Routing and data location in overlay peer-to-peer networks. Research Report RZ-3433, IBM, July 2002.

[15] Sylvia Ratnasamy, Paul Francis, Mark Handley, Richard Karp, and Scott Shenker. A scalable content-addressable network. In Proceedings of ACM SIGCOMM, September 2001.

[16] Ion Stoica, Robert Morris, David Karger, M. Frans Kaashoek, and Hari Balakrishnan. Chord: A scalable peer-to-peer lookup service for internet applications. In Proceedings of ACM SIGCOMM 2001, pages 149-160, San Diego, CA, USA, August 2001.

[17] Anthony Rowstron and Peter Druschel. Pastry: Scalable, distributed object location and routing for large-scale peer-to-peer systems. In IFIP/ACM International Conference on Distributed Systems Platforms (Middleware), pages 329-350, Heidelberg, Germany, November 2001.

[18] Ben Y. Zhao, John Kubiatowicz, and Anthony Joseph. Tapestry: An infrastructure for fault-tolerant wide-area location and routing. Technical Report UCB/CSD-01-1141, April 2001.

[19] Miguel Castro, Peter Druschel, Y. Charlie Hu, and Antony Rowstron. Exploiting network proximity in distributed hash tables. In Ozalp Babaoglu, Ken Birman, and Keith Marzullo, editors, International Workshop on Future Directions in Distributed Computing (FuDiCo), pages 52-55, June 2002.

[20] Sylvia Ratnasamy, Mark Handley, Richard Karp, and Scott Shenker. Topologically-aware overlay construction and server selection. In Proceedings of INFOCOM, June 2002.

[21] Greg N. Frederickson. Searching intervals and compact routing tables. Algorithmica, 15(5):448-466, May 1996.

[22] Robert Hinden and Stephen Deering. IP version 6 addressing architecture. Internet RFC 2373, 1998. 